\title{
A MOBILE CONTROLLED ALGORITHM FOR IMPROVING THE THROUGHPUT IN A S-ALOHA DS-CDMA SYSTEM
}

\author{
Oriol Sallent, Ramon Agustí \\ Departament de Teoria del Senyal i Comunicacions \\ Universitat Politècnica de Catalunya \\ C/ Gran Capità s/n - 08034 Barcelona - Spain \\ Tel. +34-3-401.71.97 Fax +34-3-401.72.00 \\ E-mail : oriol @ xaloc.upc.es
}

\begin{abstract}
This paper presents a novel Adaptive DS-CDMA SlottedALOHA packet random access scheme with transmitter-based spreading codes for mobiles. It is aimed at improving the throughput and message delay delivery when traffic load values below the saturation point of the conventional DSCDMA Slotted-ALOHA system are sensed in the channel. For this purpose a Mobile assisted algorithm is envisaged to control the change of the transmission rate according to the traffic load. This algorithm revealed that the optimum behavior, obtained using a Markov Chain model, may be almost reached at a low complexity cost. Moreover, priorities between mobiles could be easily established. Finally, a traffic model based on a realistic statistical length distribution of the messages illustrates how the delay delivery can be greatly reduced.
\end{abstract}

\section{INTRODUCTION}

Slotted ALOHA (S-ALOHA) has been widely recognized for packet radio applications because of both its simplicity to manage bursty traffic and its ability to deliver a shorter delay than that of fixed multiple access techniques in the presence of low traffic loads. S-ALOHA DS-CDMA scheme allows several users to transmit at the same time using a different spreading code for each one [1], [2], so that unsuccessful transmissions are caused entirely by multiple access interference, provided negligible thermal noise effects are assumed.

This paper is intended to improve the throughput achieved with a multi-receiver DS-CDMA S-ALOHA when a light load is offered to the system, and accordingly the delay decreases. For this purpose we will focus on the transmission rate used instead of the access protocol itself. In particular, different transmission rates are proposed to be used in order to utilize the most suitable rate according to the channel load at any time slot. Then, a fast and simple algorithm that command the processing gain of the Spread Spectrum DS scheme as a function of the channel load is envisaged. For low channel loads, a high CDMA processing gain is no longer necessary and it can be reduced so as to increase the actual bit rate.
The paper is organized as follows. In Section 2 an analytical model for the DS-CDMA S-ALOHA system is presented, which is used to evaluate the optimum achievable throughput with an adaptive change of transmission rate algorithm. In Section 3 a particular S-ALOHA adaptive algorithm is proposed and assessed. Section 4 considers a simple priority policy that allows different throughput levels for different groups of users. Section 5 considers a realistic situation where users generate messages that must be segmented into several packets to access the radio channel. Finally, some conclusions close the paper in Section 6 .

\section{MODEL FOR A S-ALOHA DS-CDMA ACCESS SYSTEM}

From now onwards $N$ registered users will be considered. These users can be in two different operation modes: 'origination mode' and 'backlogged mode'. In the former mode there is no packet to be retransmitted and new packets are generated with probability $p_{o}$. Terminals enter the backlogged mode when an attempt to transmit a new packet fails. In this mode, the retransmission of the backlogged packet occurs in any given slot with probability $p_{r}$. While in the backlogged mode the user does not generate any new packet.

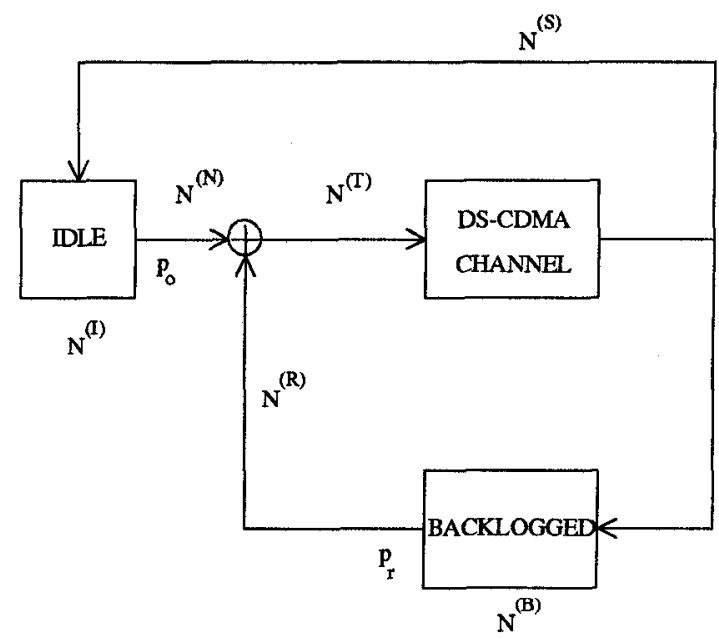

Fig. 1. Model for a S-ALOHA DS-CDMA system. 
Let $N_{k}^{(B)}$ denote the number of backlogged users at the beginning of the $k^{\text {th }}$ slot. The process defined by $N_{k}^{(B)}$ $k=0,1, \ldots$ is a Markov chain, whose performance has been studied among others in $[3,4]$. When considering DS-CDMA, the formulation must be modified as it was done in [5]. The same notation is followed in this paper, and it is summarized in Table 1 and Fig. 1.

Table 1. List of parameters for the S-ALOHA DS-CDMA model.

\begin{tabular}{|c|c|}
\hline$N$ & Number of mobiles in the system \\
\hline$N_{k}^{(B)}$ & Number of mobiles in B-mode at start of slot $k$ \\
\hline$N_{k}^{(l)}$ & Number of mobiles in I-mode at start of slot $k$ \\
\hline$N_{k}^{(T)}$ & Number of mobiles transmitting in slot $k$ \\
\hline$N_{k}^{(R)}$ & Number of mobiles in B-mode transmit in slot $k$ \\
\hline$N_{k}^{(N)}$ & Number of mobiles in I-mode transmit in slot $k$ \\
\hline$N_{k}^{(S)}$ & Number of packets correctly received in slot $k$ \\
\hline
\end{tabular}

Throughput measurements can be obtained from the markovian model considering that

$$
S=\sum_{n=0}^{N}\left(\sum_{s=0}^{n} s \times P\left\{N^{(S)}=s N^{(T)}=n\right\}\right) \times P\left\{N^{(T)}=n\right\}(1)
$$

where $S$ is in packets per slot and, if all users employ the same modulation and transmission rate, the following expression arises

$$
P\left\{N^{(S)}=s \mid N^{(T)}=n\right\}=\left(\begin{array}{l}
n \\
s
\end{array}\right)\left[P_{c}(n)\right]^{s}\left[1-P_{c}(n)\right]^{n-s}
$$

being $P_{c}(n)$ the probability of correctly detecting a packet when $n$ users have attempted transmission in a time slot.

$P\left\{N^{(T)}=n\right\}$ in (1) stands for the equilibrium distribution of the composite packet arrivals in a time slot, which could be reached in terms of the equilibrium distribution of the Markov chain. The complete derivation of the expression for this probability can be found in [5].

\section{A. S-ALOHA DS-CDMA ACCESS SYSTEM}

A BPSK DS-CDMA access system with a processing gain given by $G_{p}$ is considered. All users have been assigned random PN signature sequences. A perfect power control capable of mitigating fadings of the channel is introduced. With a view to achieving this, a continuous link between BS and MS is needed so that the mobile transmits at rate $v$ when there is information to be sent and at rate $v^{\prime}$ (in general much lower) when the terminal is not active. $v^{\prime}$ should be high enough to allow the fading on the uplink path to be tracked by updating transmitted power in response to the BS commands.
An instantaneous power control permits a huge improvement in the system performance when compared with an open loop power control, and it is, in fact, considered in the already operative [7] and the proposed CDMA systems [8] respectively. Although an instantaneous power control could be envisaged for packet radio on a packet by packet basis [9], we have retained the continuous link approach because in this case no synchronization overhead at the beginning of time slot would be required, since the mobile is already synchronized. Whether continuous power control is retained or not it is in any case irrelevant for the proposed S-ALOHA scheme taken for granted that a throughput decrease should be considered in the non-continuous packet based power control approach.

\section{B. S-ALOHA DS-CDMA PERFORMANCE}

By assuming an ideal instantaneous power control, the channel can be seen as an AWGN if we use the gaussian hypothesis to model the interference originated by other users [10], [2]. Under these conditions the following expressions hold for the evaluation of the BER

$$
P_{b}(n)=Q\left(\sqrt{2 \frac{E_{b}}{N_{o}}}\right) ; \frac{E_{b}}{N_{o}}=\frac{1}{\frac{2(n-1)}{3 G_{p}}+\frac{2(N-n)}{3 G_{p}} \frac{v^{\prime}}{v}}
$$

where $n$ is the number of simultaneous users. The probability of detecting correctly a packet containing $\alpha B$ bits is

$$
P_{c}(n)=\left[1-P_{b}(n)\right]^{\alpha B}
$$

Using the same bandwidth, to transmit at double rate $(2 v$ bits/s, where $v$ is the basic rate) half the spread capacity should be given up, thus allowing a processing gain of $G_{p} / 2$, or $G_{p} / 4$ if we transmit at $4 v \mathrm{bits} / \mathrm{s}$. However, in exchange for this reduction in processing gain, $2 B$ or $4 B$ bits per time slot can be allocated instead of only $B$.

The achievable throughput with each one of the transmission rates obtained with the previous Markov model can be seen in Fig. 2, where $G$ stands for the offered load -average number of attempted transmissions per time slot-, and $p_{o}$ is set equal to $p_{r}$ so that $p_{o}=p_{r}=p=G / N$. It is clear from Fig. 2 that the high rate $(4 v)$ is interesting when the system is lightly loaded since more bits per packet can be sent through the channel, due to the fact that the little interference observed permits a correct transmission. When the offered load increases so does the interference level, and therefore the rate $4 v \mathrm{bits} / \mathrm{s}$ is no longer interesting because of the higher BER if it is compared to the medium rate $(2 v \mathrm{bits} / \mathrm{s})$. Using $2 v \mathrm{bits} / \mathrm{s}$ in this range is better than $4 \mathrm{v}$ bits/s in the sense that a higher throughput is achieved although the packet contains half the number of bits per packet. Even for higher offered loads the same trade-off appears between medium rate $(2 v$ bits $/ s)$ and low rate $(v$ bits $/ s$ ). Transmission rate could be increased over $4 v$ bits $/$, 
but the marginal gain is each time lower as Fig. 2 shows. So, for the sake of brevity only three rates $v, 2 v$ and $4 v$ bits/s will be considered in the sequel.

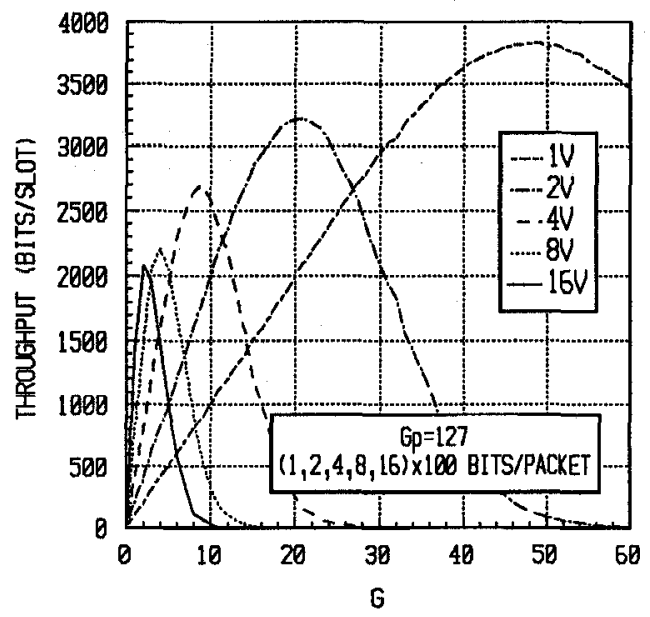

Fig. 2. Throughput performance for different transmission rates.

\section{ADAPTIVE S-ALOHA DS-CDMA}

In the light of the above results, an algorithm able to change the transmission rate used by the MS as a function of the traffic load of the system could be foresee so that the maximum possible throughput could always be obtained.

The optimum throughput attained can be analytically obtained from the above Markov model, and this result can be later used as a reference to assess the performance of the transmission rate change algorithm proposed below. In order to attain this optimum performance, the best combination of transmission rates should be obtained provided that $n$ simultaneous users are present. The resulting optimal table would be reached after an exhaustive search, as it is further explained in [6].

Since the same channel is shared by users transmitting at different rates, it should be considered that for keeping the same recieved $E_{b}$ the power received for the rate $v b i t s / s$ is half of that required for $2 v \mathrm{bits} / \mathrm{s}$ and a quarter of that needed with $4 v \mathrm{bits} / \mathrm{s}$. Consequently, the probability of getting $s$ successful packets over $n$ transmitted packets is given now by

$$
\begin{aligned}
& p\left\{N^{(S)}=s \mid N^{(T)}=n\right\}=\sum_{i=\max \left(0, s-n^{2 v}-n^{4 v}\right)}^{\min \left(n^{v}, s\right)} \sum_{j=\max \left(0, s-i-n^{4 v}\right)}^{\min \left(n^{2 v}, s-i\right)}\left(\begin{array}{c}
n^{v} \\
i
\end{array}\right)\left[P_{c}^{v}\right]^{i}\left[1-P_{c}^{v}\right]^{n^{v}-i} \\
& \left(\begin{array}{c}
n^{2 v} \\
j
\end{array}\right)\left[P_{c}^{2 v}\right]^{j}\left[1-P_{c}^{2 v}\right]^{n^{2 v}-j} \\
& \cdot\left(\begin{array}{c}
n^{4 v} \\
s-i-j
\end{array}\right)\left[P_{c}^{4 v}\right]^{s-i-j}\left[1-P_{c}^{4 v}\right]^{n^{4 v}-s+i+j}
\end{aligned}
$$

instead of (2), which applies when all users employ the same transmission rate. In turns, it is assumed that optimal combination $\left(n^{v}, n^{2 v}, n^{4 v}\right)$ is available for any $n$ and users are in fact using it. That is, $n$ is readily associated to a $\left(n^{v}, n^{2 v}, n^{4 v}\right)$ set, and so $P\left\{N^{(S)}=s / N^{(T)}=n\right\}$ is evaluated considering all possible cases giving $s$ correctly-received packets when $n^{v}$ users transmit at low rate, $n^{2 v}$ at medium and $n^{4 v}$ at high, leading to (5). Thus, the modified throughput expression is

$$
\begin{aligned}
& S=\sum_{n=0}^{N}\left[\sum_{i=\max \left(0, s-n^{2 v}-n^{4 v}\right)}^{\min \left(n^{v}, s\right)} \sum_{j=\max \left(0, s-i-n^{4 \nu}\right)}^{\min \left(n^{2 v}, s-i\right)}[B i+4 B(s-i-j)]\right. \\
& \left(\begin{array}{c}
n^{\nu} \\
i
\end{array}\right)\left[P_{c}^{\nu}\right]^{i}\left[1-P_{c}^{\nu}\right]^{n^{v}-i}\left(\begin{array}{c}
n^{2 \nu} \\
j
\end{array}\right)\left[P_{c}^{2 \nu}\right]^{j}\left[1-P_{c}^{2 v}\right]^{n^{2 v}-j} \\
& \left.\cdot\left(\begin{array}{c}
n^{4 v} \\
s-i-j
\end{array}\right)\left[P_{c}^{4 v}\right]^{s-i-j}\left[1-P_{c}^{4 v}\right]^{n^{4 v}-s+i+j}\right] \times P\left\{N^{(T)}=n\right\} \quad \text { bits/slot }
\end{aligned}
$$

where $B$ is the number of bits in a BPSK $v$ rate packet $(B=100$ bits all over this paper) and $S$ is expressed in bits/slot. Fig. 3 shows this optimum throughput in comparison with the individual behaviors.

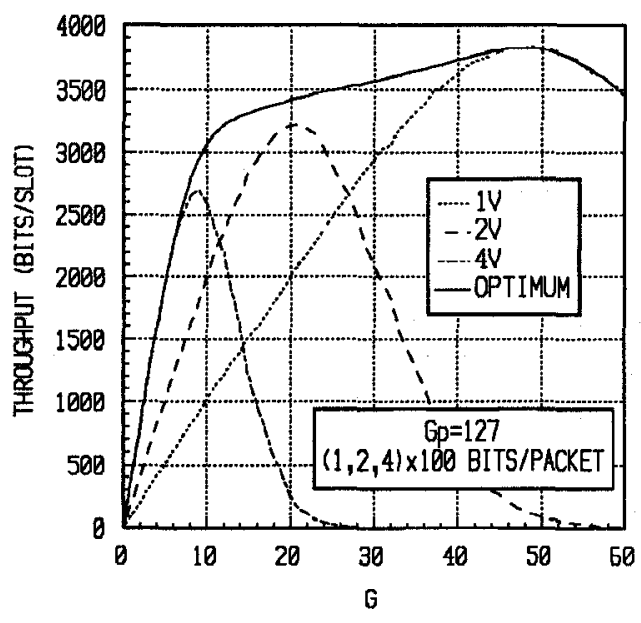

Fig. 3. The very best achievable throughput for the S-ALOHA DS-CDMA system.

\section{CHANGE OF THE TRANSMISSION RATE ALGORITHM}

In this section a proposal of an adaptive change of the transmission rate algorithm is addressed. The basic idea consist in sensing the traffic load through the channel in order to accommodate transmission rates accordingly.

The proposed simple algorithm carried out by the mobile station (MS) works as follows: each terminal traces its own evolution during the transmission time, that is, terminals count their successful and erroneous packets. In the absence of errors the mobile will assume a low traftic load and tries to use a higher transmission rate. The throughput should be increased in this way. If errors occur, the mobile decides that the channel is too loaded and tries a lower transmission rate. 
In this case fewer bits per packet are transmitted, but a global improvement of the throughput should also follow because these bits can be now detected correctly since processing gain increases accordingly.

Let us note that this decision is taken by the mobile without any exchange of information with the Base Station (BS) except for the packets acknowledgment. Even the MS does not need to indicate its choice of transmission rate before using it because the very same BS could be able to detect which one is arriving.

Specifically, the MS only needs to establish two parameters: the number of consecutive packet failures before changing to a lower rate ( $\max$ tr) and the number of consecutive packet successes before trying a higher rate (min_suc).

Fig. 4 shows the throughput attained for $\mathrm{N}=60$ registered mobiles. Fig. 1 has been taken as a reference in order to appreciate whether mobiles choose the most suitable rate or not. In spite of the simplicity of the algorithm, the envelope of the three individual graphs is almost reached, and this is not far from the optimum behavior. Results have been obtained with max tr=1 and min_suc=6, which is the best choice, once other possibilities have been studied.

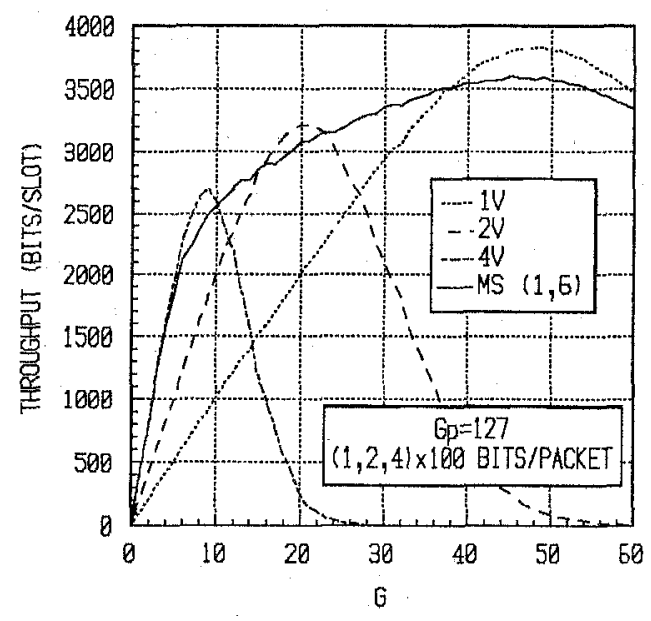

Fig. 4. Throughput performance for MS algorithm.

\section{PRIORITIES FOR THE MS ALGORITHM}

It has been pointed out earlier that some kind of priorities could be established between mobiles when applying the change of transmission rate algorithm. Since this algorithm is very simple and so is the access protocol considered, priorities assignment procedures should also be simple.

Thus, users subscribing to the system would be clasified in several categories depending on their transmission rate needs and the service capacity of the BS (which depens on transmission rate needs of previously subscribed users). The simple objective is trying to split the achievable capacity in an unequaly way, keeping the same global throughput as far as possible.
In what concerns MS algorithm, some priority could be assigned when the mobile registers in the system and receives the values of (maxtr,minsuc), which define algorithm behaviour. Results when (maxtr,minsuc) are set to $(1,6)$ and all mobiles use the same parameters have already been shown in Fig. 4. However, the BS could send more optimistic couple to those users with higher rate requirements, although it should be compensated sending more cautious parameters to the lowest priority group in order to keep the mean interference power.

A good trade-off is obtained for the case $N=60$ with 10 high priority users employing $(2,4), 40$ users with $(1,6)$ and 10 low priority mobiles using $(1,9)$. It can be seen in Fig. 5 that certain degree of priority can be assigned.

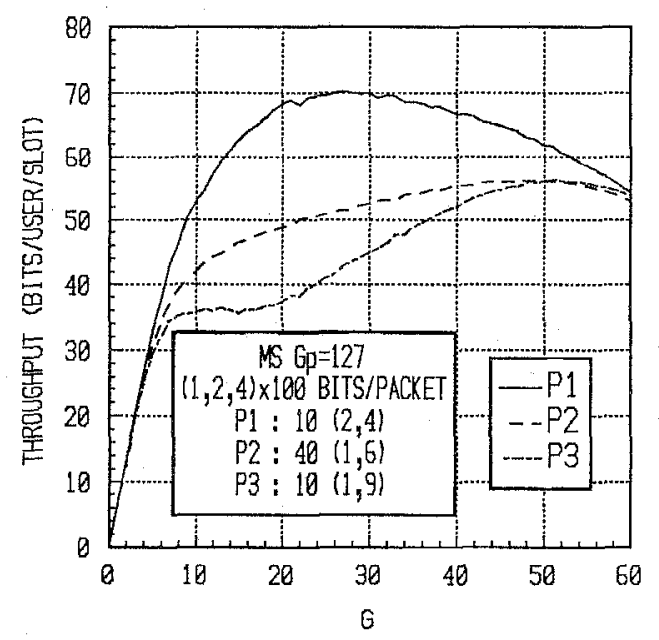

Fig. 5. Priorities for different groups with MS algorithm.

\section{MESSAGES TRAFFIC MODEL}

In a real scenario, the length of the message may not coincide with the length of a packet. If too small, it must be completed by dummy bits. If too large, it must be conveyed over several packets.

In order to assess the benefits from using the previous adaptive S-ALOHA CDMA scheme in such scenario a simulation aiming at obtaining the average message delay in terms of the throughput has been carried out. From now onwards and for simulation purposes, mobiles generating messages of mean length of 4.000 bits and an uniform distribution between 3.000 and 5.000 bits have been assumed. For simplicity we will only consider steps of 100 bits in message length. The silence periods occurring between messages are expressed in time slots and have been modeled with a uniform distribution $(0-2 \mathrm{~T})$, where $\mathrm{T}$ has been internally introduced in the simulation as a parameter. This value determines the activity factor of the sources given by one message every $\mathrm{T}$ time slots on the average $(4000 / \mathrm{T}$ bits/slot). 
In order to show some representative results, Fig. 6 plots the throughput versus the delay performance for $v, 2 v, 4 v$ rates respectively obtained for $\mathrm{N}=200$ registered users, a fixed retransmission probability $p_{r}=0.05$ and an infinite buffer size. It can be seen from Fig. 6 that for low loads an asymptotic message delay of 10 slots, corresponding to 10 consecutive successfully transmissions of 400 bits per time slot, is attained at $4 v$ bits $/ \mathrm{s}$. Asymptotic message delay of 20 time slots at $2 v$ bits/s and 40 time slots at $v$ bits/s respectively show the potential benefit that can be reached by using the adaptive transmission rate procedure described above for a CDMA S-ALOHA system.

Finally, Fig. 7 show the results obtained for mobile controlled algorithm (MS). It follows almost perfectly the best graph, until the load is so high that it becomes clear that $v$ rate should be used and the MS algorithm procedure performs useless.

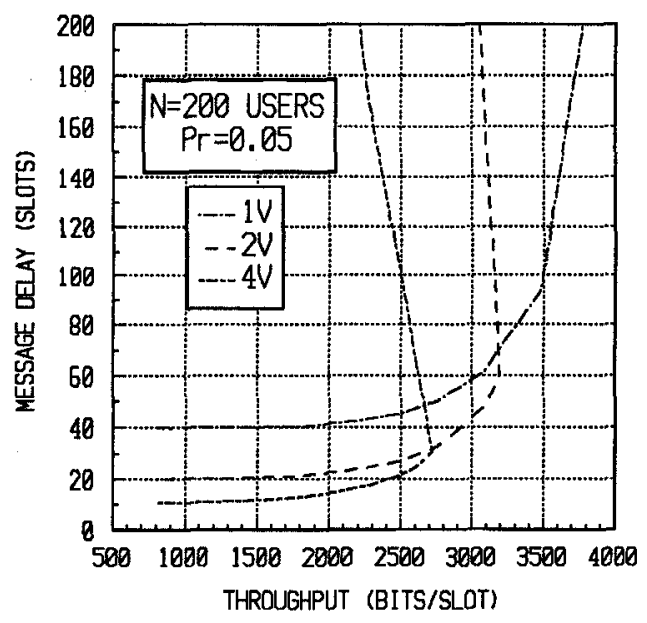

Fig. 6. Delay versus throughput behavior with messages.

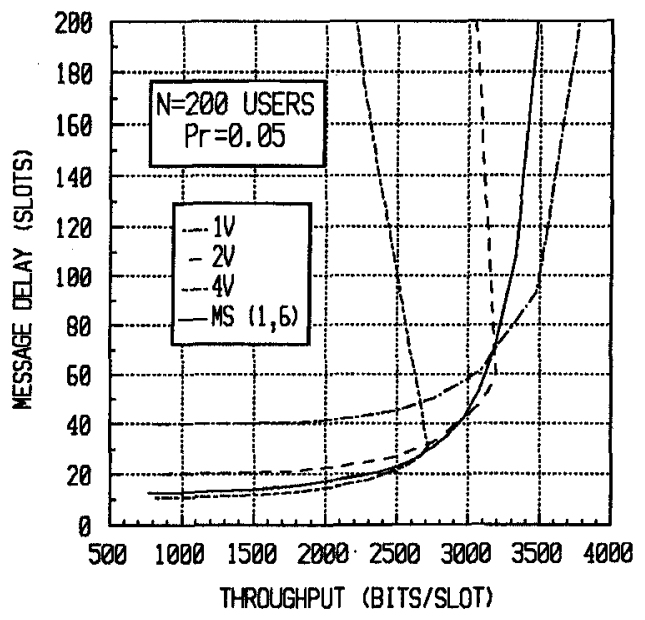

Fig. 7. Mobile controlled algorithm performance.

\section{CONCLUSIONS}

A new Adaptive DS-CDMA S-ALOHA technique for packet mobile communications access based on the choice of the most suitable transmission rate at any time slot has been addressed in this paper. The throughput and delay performance attained outperforms by far those obtained with a conventional DS-CDMA S-ALOHA scheme when the offered load is under the saturation point . In particular, this mobile assisted algorithm allows to obtain almost the maximum attainable performance at a very low complexity cost.

\section{REFERENCES}

[1] D. RAYCHAUDHURI, "Performance Analysis of Random Access Packet-Switched Code Division Multiple Access Systems", IEEE Transactions on Communications, Vol. Com. 29, No. 6, June 1981, pp. 895-901

[2] R. K. MORROW, J.S. LEHNERT "Packet Throughput in Slotted ALOHA DS/SSMA Radio Systems with Random Signature Sequences", IEEE Transactions on Communications, Vol. Com. 40, No. 4, July 1992, pp. 12231230.

[3] R. ROM, M. SIDI, Multiple Access Protocols. Performance and Analysis, Springer-Verlag, 1990.

[4] L. KLEINROCK, S.S. LAM, "Packet Switching in a Multi-access Broadcast Channel: Performance Evaluation ", IEEE Transactions on Communications, Vol. Com.-23, No. 4, April 1975, pp.410-422.

[5] Z. LIU, M. EL ZARKI, "Performance Analysis of DSCDMA with Slotted ALOHA Random Access for Packet PCNs ", Wireless Networks 1 (1995), pp. 1-16.

[6] O. SALLENT, R. AGUSTI, "A Proposal of an Adaptive S-ALOHA Access System for a Mobile CDMA Environment", submitted for publication.

[7] A. SALMASI, K. S. GILHOUSEN, "On the System Design Aspects of CDMA Applied to Digital Cellular and Personal Communications Networks", Proc. 41 $1^{\text {st }}$ IEEE Vehicular Technology Conference, St. Louis, May 1991.

[8] A. BAIER et al., "Design Study for a CDMA-Based Third-Generation Mobile Radio System ", IEEE Journal on Selected Areas in Communications, Vol. 12, No. 4, May 1994, pp. 733-743.

[9] M. J. McTIFFIN et al., "Mobile Access to an ATM Network Using a CDMA Air Interface ", IEEE Journal on Selected Areas in Communications, Vol. 12, No. 5, June 1994, pp. 900-908 .

[10] M. B. PURSLEY, "Performance Evaluation for PhaseCoded Spread-Spectrum Multiple-Access Communication Part I: System Analysis ", IEEE Transactions on Communications, Vol. Com.-25, No. 8, August 1977, pp.795799. 